\title{
Nonlinear excitations in DNA: Aperiodic models versus actual genome sequences
}

\author{
Sara Cuenda* \\ Grupo Interdisciplinar de Sistemas Complejos (GISC) and Departamento de Matemáticas, Universidad Carlos III de Madrid, \\ Avenida de la Universidad 30, 28911 Leganés, Madrid, Spain ${ }^{\dagger}$ \\ Angel Sánchez \\ Grupo Interdisciplinar de Sistemas Complejos (GISC) and Departamento de Matemáticas, Universidad Carlos III de Madrid, \\ Avenida de la Universidad 30, 28911 Leganés, Madrid, Spain \\ and Instituto de Biocomputación y Física de Sistemas Complejos, Universidad de Zaragoza, 50009 Zaragoza, Spain
}

(Received 2 July 2004; published 9 November 2004)

\begin{abstract}
We study the effects of the genetic sequence on the propagation of nonlinear excitations in simple models of DNA in which we incorporate actual data from the human genome. We show that kink propagation requires forces over a certain threshold, a phenomenon already found for aperiodic sequences [F. Domínguez-Adame et al., Phys. Rev. E 52, 2183 (1995)]. For forces below threshold, the final stop positions are highly dependent on the specific sequence. Contrary to the conjecture advanced by Domínguez-Adame and co-workers, we find no evidence supporting the dependence of the kink dynamics on the information content of the genetic sequences considered. We discuss possible reasons for that result as well as its practical consequences. Physically, the results of our model are consistent with the stick-slip dynamics of the unzipping process observed in experiments. We also show that the effective potential, a collective coordinate formalism introduced by Salerno and Kivshar [Phys. Lett. A 193, 263 (1994)], is a useful tool to identify key regions in DNA that control the dynamical behavior of large segments. As a side result, we extend the previous studies on aperiodic sequences by analyzing the effect of the initial position of the kink, leading to further insight on the phenomenology observed in such systems.
\end{abstract}

DOI: 10.1103/PhysRevE.70.051903

PACS number(s): 87.14.Gg, 87.17.Aa, 05.45.Yv, 87.15.La

\section{INTRODUCTION}

Nonlinear models are becoming widely used to capture and understand emergent phenomena in complex systems [1]. The pioneering field in this direction was physics, where nonlinear phenomena have been the subject of intense study since the seminal work of Fermi, Pasta, and Ulam almost 50 years ago [2]. The success of this approach in modeling complex systems led scientists in other fields to apply it to their own research. That is the case in biology, where nonlinear models were successfully introduced more than 20 years ago [3] (see also [4] for references). One of the subjects where nonlinear models have been more productive is the modeling of DNA physics [5-9], which began in 1980 with the model proposed by Englander and co-workers [10]. Since then, a lot of work has been devoted to nonlinear excitations in DNA, both from the dynamics and the statistical mechanics viewpoints. We refer the reader to [7,9] for historical accounts and extensive summaries of the available results, among which we want to highlight the Peyrard-Bishop model [11] (see also the generalizations and improvements proposed in $[12,13])$. In spite of its simplicity, this nonlinear lattice system can accurately (sometimes even quantitatively) reproduce the phenomenology experimentally observed in DNA (see, e.g., [14]).

\footnotetext{
*Electronic address: scuenda@math.uc3m.es

${ }^{\dagger}$ URL: http://gisc.uc3m.es

"Electronic address: anxo@math.uc3m.es
}

Most of the research done in the framework of the abovementioned models refers to homopolymers, i.e., homogeneous DNA molecules consisting entirely of A-T or C-G base pairs. One problem of special interest was the thermal denaturation transition, which takes place at temperatures around $90^{\circ} \mathrm{C}$ when the two strands of the DNA molecule separate. Having understood this transition quite well $[9,15]$, the main focus of research has been on dynamical and transport features. Additional motivation for such a shift arises from the capability to carry out experiments on single molecules, achieved in the last few years [16]. Another particularly important, related question is genomics, or gene identification, which is profiting enormously from the information obtained from dynamical models [17]. Of course, these applications require unavoidably the study of DNA heteropolymers, where the distribution of A-T and C-G base pairs follows nonuniform, nonhomogeneous sequences obtained from genome analysis. In this context, physical models are also being used to identify dynamically relevant sites, such as promoters [18] in short genomes (e.g., viral). Further advance along this line requires good models for DNA dynamics that can be computationally efficient, in order to treat much longer sequences. Such models will also be of use to achieve a better understanding of the relationship between sequence, physical properties, and biological function.

In this work, we address these issues in the framework of a very simple model, namely the Englander model [10], which we generalize by incorporating actual genetic sequences. Our aim is to assess whether the so-modified model, in spite of its simplicity, reproduces the important features of DNA molecule dynamics accurately enough to be 


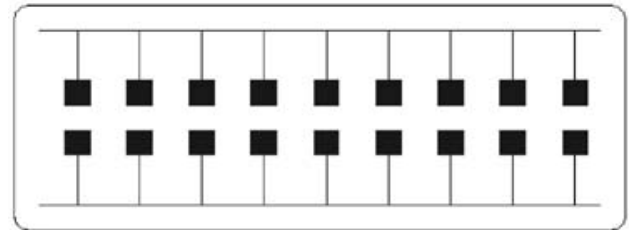

(a)

(b)

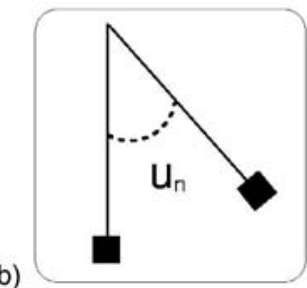

FIG. 1. Sketch of the Englander model. The two sugarphosphate backbones of the DNA strands are depicted as two straight lines connected by the base pairs (bases are the squares). The lower strand is assumed to be fixed, and as shown on the right, the angle $u_{n}$ is the deviation of the upper base of the $n$th pair with respect to the plane defined by the fixed base pairs in the lower strand.

useful for genomic and related applications. We will also discuss an approximation in terms of an effective potential and its application to understanding the dynamics of nonlinear excitations in DNA. In addition, we will test a conjecture, put forward by Domínguez-Adame and co-workers [19], that the dynamics of nonlinear excitations along DNA molecules should, at the level of the Englander model, depend on the information content of the chain. To this end, the paper is organized as follows. In Sec. II, we review the Englander model and the previous attempts to include inhomogeneity or genetic information in it. In so doing, we will discuss in some detail the results of Domínguez-Adame et al., extending their work to consider the effects of changing the initial location of the propagating kinks. Subsequently, in Sec. III, we present our results on the Englander model with human genome sequences. We analyze the results by comparing them to the phenomenology observed on aperiodic sequences and to the experimentally observed DNA dynamics. Finally, Sec. IV concludes the paper by summarizing our main results and their possible implications.

\section{BACKGROUND}

\section{A. Model definition: The homogeneous case}

The model proposed by Englander et al. [10] is sketched in Fig. 1, where the dynamics of one of the strands of the DNA is represented as a chain of pendula; leaving the other strand fixed (the one at the bottom in the picture), the base pairs of the (upper) strand behave like pendula in an effective "gravitational field" caused by the tendency of the base pairs of the two strands to be facing each other. It must be realized that this model describes only the dynamics of the base pairs around the sugar-phosphate backbone, which is assumed to be fixed as well. Introducing damping and an external force, these pendula are described by the discrete, dc-driven, damped sine-Gordon model, given by

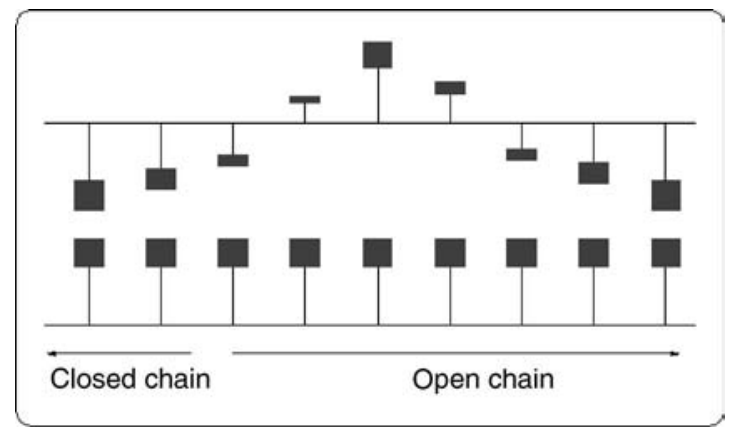

FIG. 2. Kink soliton in the sine-Gordon model. The kink joins a sector of the chain where bases are closed, $u_{n}=0$, to another one where bases have performed a complete turn, $u_{n}=2 \pi$. In this last part, the chain is said to be open if the model is to represent mechanical denaturation.

$$
\ddot{u}_{n}-\frac{1}{a^{2}}\left(u_{n+1}-2 u_{n}+u_{n-1}\right)+q_{n} \sin u_{n}=-\alpha \dot{u}_{n}-F,
$$

where $a$ is the lattice spacing, $q_{n}$ is a site-dependent constant which arises from the specific parameters for the pendulum at site $n, \alpha$ is the damping coefficient, and $F$ is a driving term possibly acting on the chain. We note at this point that Eq. (1) reflects mainly the difference in interactions between A-T and $\mathrm{C}-\mathrm{G}$ base pairs, but not other differences such as in mass or moments of inertia. It should also be clear that many other relevant factors (geometry, inhomogeneous stacking interactions [20]) have also been neglected, in the spirit of designing a model as simple as possible that still behaves like DNA molecules.

When $q_{n}=q$ (i.e., we have a homopolymer) in Eq. (1) and the lattice spacing is very small, the system of ordinary differential equations (1) can be very well approximated by its continuum limit. Letting $u_{n}(t) \rightarrow u(x, t)$ when $a^{2} \ll q$, we find the driven, damped sine-Gordon equation,

$$
\partial_{t}^{2} u-\partial_{x}^{2} u+q \sin u=-\alpha \partial_{t} u-F .
$$

It is well known [1] that, in the absence of force, i.e., $F=0$, Eq. (2) possesses soliton solutions of the kink type, whose expression is

$$
\phi_{ \pm}(x, t)=4 \arctan \left\{\exp \left[ \pm \sqrt{q}\left(\frac{x-x_{0}-v t}{\sqrt{1-v^{2}}}\right)\right]\right\},
$$

where the plus or minus sign stands for kinks or antikinks, respectively, and $0 \leqslant v \leqslant 1$ represents their velocity. In the case $F=0$ and in the presence of damping, $\alpha \neq 0$, the only possible value for the velocity is $v=0$. When both damping and force are present, the balance between the two effects leads to kinks propagating at a constant, nonzero velocity. An analytical expression for that velocity can be easily derived [again, for homogeneous sequences, i.e., from Eq. (2)] from energy-conservation arguments (see [21]),

$$
v=\left[1+q\left(\frac{4 \alpha}{\pi F}\right)^{2}\right]^{-1 / 2} \text {. }
$$

Figure 2 depictes the physical meaning of a kink solution in the context of DNA modeling: the bases of the upper chain 
perform a complete, smooth turn around the sugar-phosphate backbone, from $u_{n}=0$ to $u_{n}=2 \pi$.

One feature of the model that deserves some comment is its mesoscopic character. Experiments and a large part of the theoretical work done so far on mechanical denaturation or unzipping ([16]; for the first unzipping experiments, see [22]) deal with forces acting on the last base of one of the strands pulling them apart. In the perspective of Englander's model, we are not trying to describe accurately the way the chain is pulled but rather we are looking at how this pulling affects the "propagation" of the opening. Of course, both approaches are different and need not yield the same results, although one expects in principle that the qualitative behavior should be the same. As we will see below, the advantage of our approach is that it allows some analytic insight into the problem, much less amenable from the microscopic viewpoint.

\section{B. Inclusion of inhomogeneities}

The results we have summarized in the previous subsection refer mostly to the homopolymer case, which was the one initially considered by Englander $e t$ al. [10]. As stated in the Introduction, our main goal is to understand the effects of inhomogeneity, particularly of genetic sequences obtained from real data. We stress that including inhomogeneity, i.e., $q_{n}$ depending on $n$ in Eq. (1), renders the theoretical approach in the preceding subsection inapplicable. To our knowledge, DNA sequences from living organisms were first studied in the framework of the Englander model by Salerno, who considered the effects of the sequence by studying spontaneously traveling kinks along the $\mathrm{T} \mathrm{A}_{1}$ promoter (a region of the genome preceding a gene where the transcription activity starts) of the bacteriophage T7. Salerno studied further other regions of the bacteriophage [24], finding that the dynamical activity of kinks should be very special in the promoters. Recently, the problem was revisited in [25] following the sequencing of the whole genome of the bacteriophage T7. The main conclusion of this work was that there is indeed a significantly higher degree of activity in promoter regions, even in the presence of noise.

The results of the simulations reported in $[23,24]$ were analyzed theoretically by Salerno and Kivshar [26], who, using a collective coordinate approach (see, e.g., [27] for a review on collective coordinate techniques for solitonbearing equations), developed a description of the kink dynamics in terms of an effective potential. We will not review in detail the procedure to obtain this effective potential but, instead, we will simply outline the main steps. The idea is to consider the undamped model, i.e., Eq. (1) with $\alpha=0$, on a finite lattice of $N$ sites, described by the Hamiltonian

$$
\begin{aligned}
H^{s G}[\{u\}]= & \sum_{n=1}^{N}\left\{\frac{1}{2} \dot{u}_{n}^{2}+\frac{1}{2 a^{2}}\left[u_{n+1}-u_{n}\right]^{2}+q_{n}\left(1-\cos u_{n}\right)\right. \\
& \left.+F u_{n}\right\} .
\end{aligned}
$$

We now take the following ansatz:

$$
\phi_{n}(X(t))=4 \arctan \left(\exp \left\{\sqrt{q_{\mathrm{avg}}}[n a-X(t)]\right\}\right),
$$

where $q_{\text {avg }}$ is the average value of $q_{n}$ over the chain, and $X(t)$ is a collective coordinate variable which stands for the center of the kink. Substituting this expresion into the Hamiltonian (5), we arrive at

$$
E_{\text {eff }}=\frac{1}{2} \dot{X}^{2}+V_{\text {eff }}\left(n,\left\{q_{n}\right\}\right),
$$

which is formally equivalent to the energy of a particle subjected to the action of the effective potential $V_{\text {eff }}$. The exact formula for this potential is quite cumbersome and we do not need to reproduce it here. We refer the reader interested in the details of the calculation and the full result to $[19,26,28]$.

\section{Aperiodic chains}

Since the sequences considered by Salerno [23,24] are very small, the question arises immediately as to the real relevance of those results. On the other hand, in 1995 there were not many genomic data available, making the question difficult to answer. To overcome this difficulty, DomínguezAdame and co-workers [19] proposed to mimic the behavior of DNA heteropolymers of biological relevance by replacing the sequence-dependent values $q_{n}$ by an aperiodic, but fully deterministic sequence. The rationale behind this choice is to consider the DNA sequence as nonperiodic but also nonrandom, in so far as it carries information. As an example, they considered the Fibonacci and Thue-Morse sequences. Most of the results were related to the former, which is generated according to the rules $q_{a} \rightarrow q_{a} q_{b}, q_{b} \rightarrow q_{a}$ repeteadly applied to the initial seed $q_{a}$.

Being the immediate predecessor of the present work, it is worth summarizing here the results in [19]. As a first step, Domínguez-Adame et al. constructed a periodic sequence $\left\{q_{n}\right\}$ whose unit cell was a Fibonacci sequence of order $k$, $F_{k}\left(F_{1}=q_{a}, F_{2}=q_{a} q_{b}, F_{3}=q_{a} q_{b} q_{a}, \ldots\right)$. This amounts to saying that the resulting chain is given by $\left\{q_{n}\right\}=F_{k} F_{k} F_{k} \ldots$. The number of unit cells was chosen such that the length of the whole sequence $\left\{q_{n}\right\}$ was about 4000 sites. Subsequently, they placed a kink of the form (6) at rest in the middle of the sequence and pulled it with a constant force $F$ in order to find the asymptotic velocity of the kink [recall that $\alpha \neq 0$ in Eq. (1), implying the existence of damping]. This computation was performed for various orders (i.e., sizes) of the Fibonacci unit cells, $F_{k}$, up to a nonperiodic, full Fibonacci sequence of 4181 sites $(k=18)$. The results of DomínguezAdame et al., subsequently confirmed in [28], pointed out the existence of an intriguing phenomenon, namely the existence of a threshold force for a kink to start moving along the chain. Once the force is above the threshold, the kink moves asymptotically with a periodic velocity, as a consequence of the periodicity of the constructed sequence (except in the case of the aperiodic $F_{18}$ sequence, in which the asymptotic, aperiodic velocity fluctuates around a mean value), given by the balance of damping and driving. Although the original derivation of Eq. (4) is valid for a homogeneous chain, reproducing it with a value $q_{\text {avg }}$, obtained by averaging $q_{n}$ over all lattice sites leads to a prediction which is very accurate, 


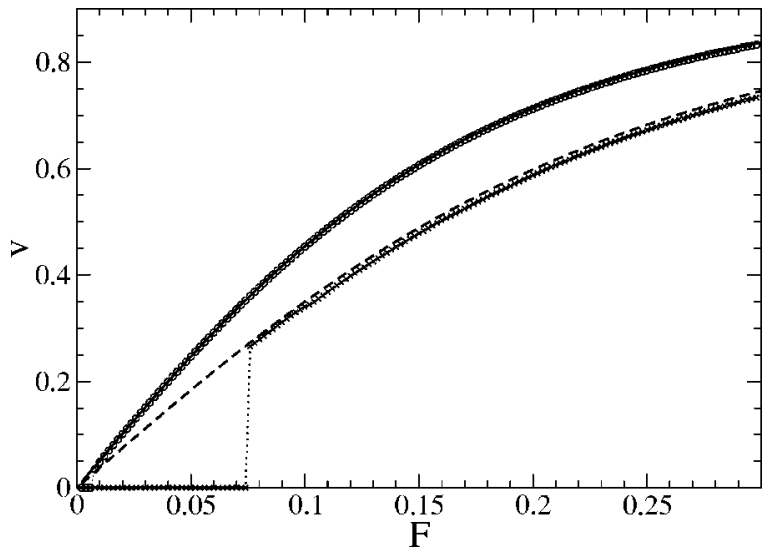

FIG. 3. Steady-state velocity vs applied force for a periodic Fibonacci chain. The lines correspond to the theoretical approach and the points to numerical simulations, both for two periodic chains with unit cell $F_{9}$, of 55 sites, but with different values of $q_{a}$ and $q_{b}:(\times) q_{a}=1$ and $q_{b}=10$, theoretical approach with $q_{\mathrm{avg}}$ $=4.436 ;(\bigcirc) q_{a}=2, q_{b}=3$, theoretical approach with $q_{\text {avg }}=2.382$.

above the threshold, for all the periodic chains, including the final, full Fibonacci chain $F_{18}$. Such a prediction overestimates slightly the velocity when increasing the ratio between the two parameters $q_{a}$ and $q_{b}$ (see Fig. 3 for details). In addition, in [19], it was also found that the threshold value depended on the length of the periodic chain considered: Increasing the size of the unit cell $F_{k}$ led to an increasing of the threshold, reaching a limiting value for the full Fibonacci sequence.

Based on the above summarized results and on the fact that, for the same parameters, random chains exhibited much larger threshold values (around $F \simeq 0.5$ ), Domínguez-Adame et al. concluded that long-range-order effects give rise to measurable consequences on kink dynamics in aperiodic chains. Further, they suggested that the fact that there are long-range correlations in DNA and, in any event, that it contains information and is not purely random, could lead to similar phenomenology in the propagation of nonlinear coherent excitations along the molecule. At this point, we revisit this question, benefiting from the huge body of genome data available nowadays. As we will show in the next section, on the grounds of the revision presented here, the conjecture about the information in [19] is likely to be incorrect, at least if we consider that the information content of coding DNA is different from noncoding DNA. We will come back to this issue in the Conclusions, after discussing the results for real DNA sequences in the next section. Before proceeding to those results, we find it interesting to extend the results in [19] by discussing the relevance of the choice of the initial location for the kink. This we do in the subsection immediately below.

\section{Effect of the initial position of the kink}

As we have said above, Domínguez-Adame et al. considered only the existence of a threshold force for propagation on aperiodic chains. Although this phenomenon was addressed and interpreted correctly in [19], we have found that

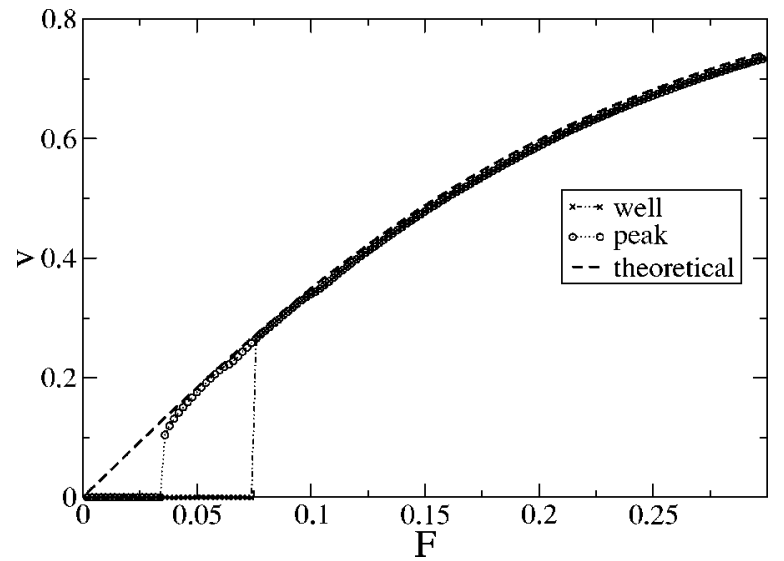

FIG. 4. Numerical results of steady-state velocity vs applied force for a periodic Fibonacci chain with unit cell $F_{9}, q_{a}=1$ and $q_{b}=10$, but starting from a peak $(\bigcirc)$ and from a well $(\times)$. The corresponding threshold force of the simulation starting from a well is larger than the one starting from a peak.

depending on the shape of the effective potential around the starting position of the kink, it is more or less difficult to make it move until the end of the lattice. If the initial position of a kink is a peak of the effective potential, it will cost less force to overcome the next barrier than if the starting point is a well, as shown in Fig. 4.

Given the above considerations, one can expect that for sequences formed with unit cells of different sizes, but with an identical effective potential in the vicinities of the starting points, the threshold force will be the same in both cases. This can be seen in Fig. 5: In Figs. 5(a) and 5(b), we see that for small unit cells, the main structure of peaks and wells of the Fibonacci sequence has not been formed yet. This can be seen, for instance, in the effective potentials of sequences with unit cell given by $F_{7}$ (of size 21) and larger, which have the same values in the surroundings of the beginning of each unit cell. If we choose the initial position of a kink to be near the beginning of a unit cell, the force needed to overcome the first barrier will be the same as in another sequence with a different unit cell, if the initial positions, related to the beginning of each unit cell, are the same. For unit cells of smaller size, the effective pottential is different and, therefore, so is the threshold force [see Fig. 5(c)].

\section{EFFECTS OF THE SEQUENCE}

We now turn to the question we posed above, namely the effects of inhomogeneity arising from the genetic sequence on the kink propagation. To this end, we have simulated numerically Eq. (1) with $\left\{q_{n}\right\}$ sequences obtained from the human genome. We want to stress that this description of DNA, arising from the original model of Englander and coworkers [10], intends to be only qualitatively correct. Therefore, the parameters can be freely chosen, trying, of course, to mimic the real ones. Accordingly, we choose $q_{a}=2$ and $q_{b}=3$ depending on whether we have at site $n$ an A-T pair, linked by two hydrogen bonds, or a C-G pair, with three hydrogen bonds. The other parameters are chosen as in [19], 

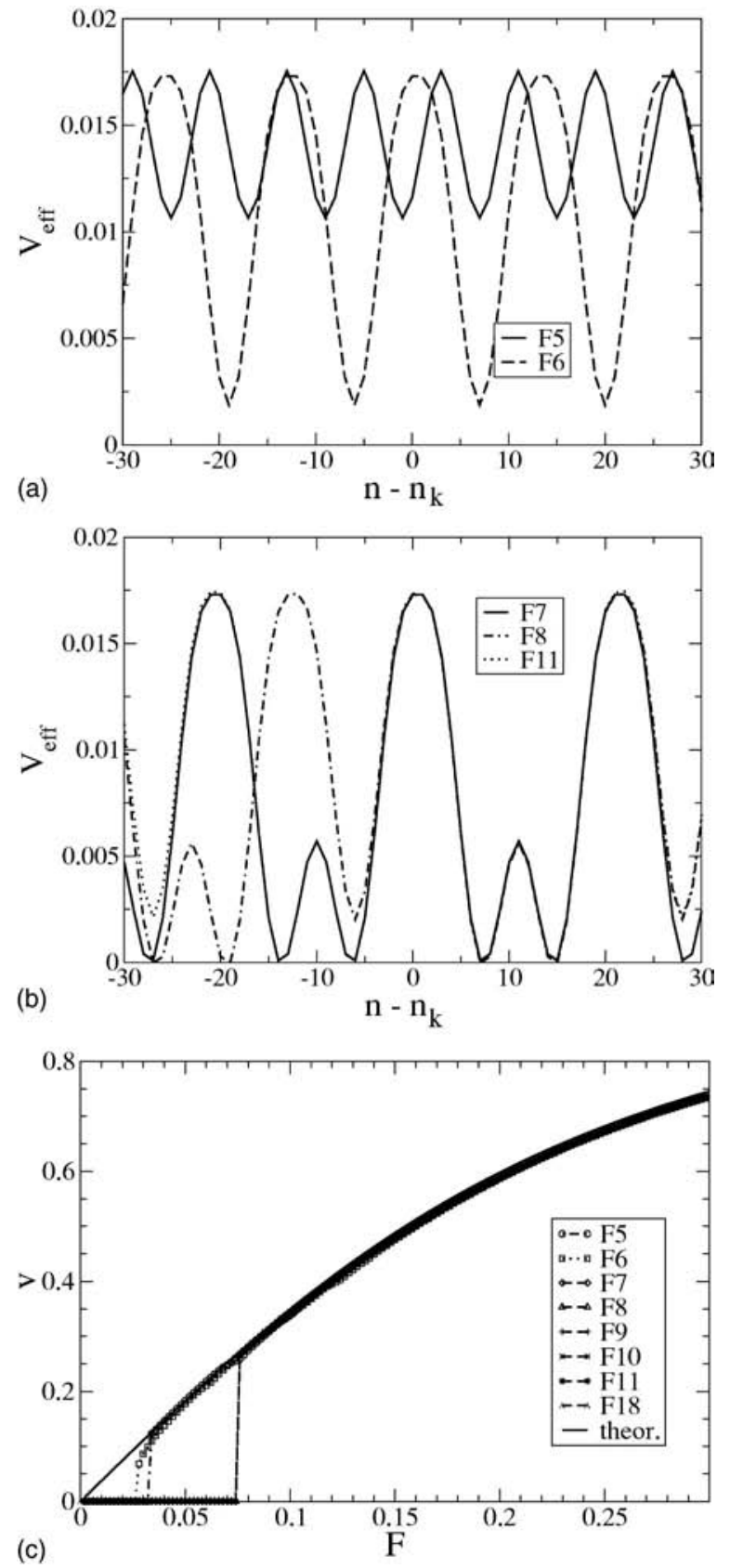

FIG. 5. (a) and (b) Effective potential around the beginning of a unit cell. There is a translation $n_{k} \equiv\left|F_{k}\right|$ in $n$ for each sequence constructed with $F_{k}$ 's in order to see the shape of the curve in the surroundings of the beginning of a unit cell within each type of sequence. There is another vertical shift to put the maxima of the different curves at the same level. For clarity, the plots for $k=5,6$ are shown in (a), and those for $k=7,8,11$ are shown in (b). (c) Steady-state mean velocity versus applied force for chains with different orderings and parameters $q_{a}=1$ and $q_{b}=10$. The line corresponds to theoretical asymptotic velocity for $q_{\mathrm{avg}}=4.438$, which is the averaged value of an infinite Fibonacci sequence, $F_{\infty}$. The points correspond to the numerical results for different periodic chains with unit cell $F_{k}$.
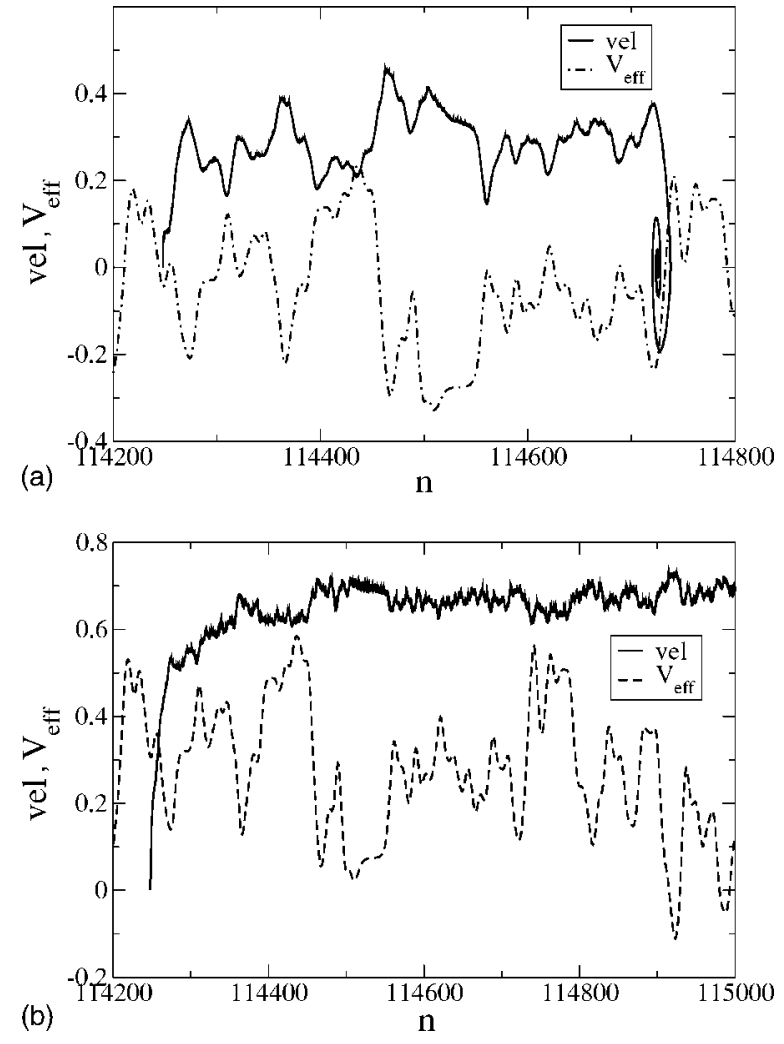

FIG. 6. Simulations of the kink soliton in the sine-Gordon model with genome data. Shown are the velocity (solid line) and the (properly scaled to fit in the plot) effective potential (dashed line) vs the position along the chain. Up: $F=0.06$; the kink travels along the sequence but stops at an effective potential well. Down: $F=0.07$; the kink travels along the whole chain. The DNA sequence corresponds to contig NT_028395.2 of human chromosome 22, between positions 114100 and 115100 , part of a gene.

namely, lattice spacing $a=0.1$ and damping $\alpha=0.1$. With respect to the lattice spacing, we want to point out that the chosen value leads to a width of the kink, which is comparable to that of spontaneous openings of real DNA chains (some 20-40 base pairs). The numerical simulations have been carried out with a standard fourth-order Runge-Kutta scheme, with free or periodic boundary conditions. Finally, for the sequences we use data obtained from the National Center for Biotechnology Information for the human genome [29].

Typical results from our simulations are plotted in Fig. 6, which presents a phase-space plot for the center of the kink on an actual DNA sequence when the kink is forced with two different drivings. For reference, we plot in the same graph the effective potential, which can be computed for any sequence as described in the previous section. It is important to note that the effective potential does not contain the contribution of the force $F$, and therefore its most relevant information is the position of the peaks and valleys. With this in mind, we see that the dynamics occurs basically as in the case of the Fibonacci chain. There is a threshold for the kink to propagate along the whole chain, shown by the fact that the kink ends up trapped at a potential well in the upper plot of Fig. 6, although the effective potential structure is very 

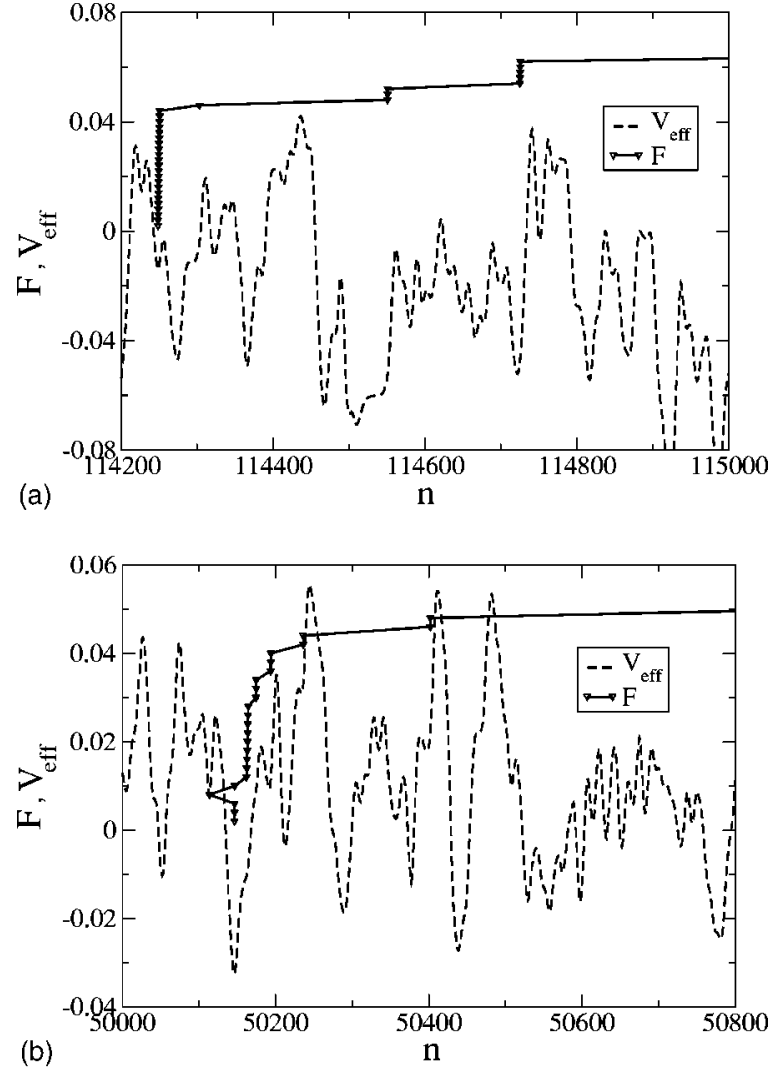

FIG. 7. Simulations of the kink soliton in the sine-Gordon model with genome data. Shown are the force (solid line) required to reach a certain position and the (properly scaled to fit in the plot) effective potential (dashed line) vs the position along the chain. Thresholds can be seen as the maximum force values in each plot. Comparison of propagation along a coding region (up, same contig as in Fig. 6, positions 114000 to 115000 ) and a noncoding region (down, same contig as in Fig. 6, positions 50000 to 51000 ).

different from the Fibonacci case. When the applied force exceeds the threshold, the kink can propagate along the whole chain (lower plot), with a velocity which is again a (fluctuating) balance of damping and forcing.

Figure 7 addresses one of the main issues we want to discuss: the relevance of the information content for the existence and characteristics of the threshold force. To this end, it collects our observations regarding the existence of thresholds in two examples: a coding region and a noncoding region. We again see the existence of threshold forces, in agreement with a description in terms of an effective potential. However, we do not observe any qualitative or otherwise relevant difference between the kink dynamics (or the effective potential) in the two regions. This is the case with all the regions we have analyzed. Therefore, the conjecture by Domínguez-Adame et al. based on their Fibonacci results that information may lead to different kink dynamical properties is, at least at the level of our simple model, not in agreement with the simulation results. Of course, this is one example; although similar results are obtained for different coding and noncoding sequences, we cannot exclude that the threshold behavior is statistically different in the two types of regions. However, from our simulations we consider this possibility quite unlikely.
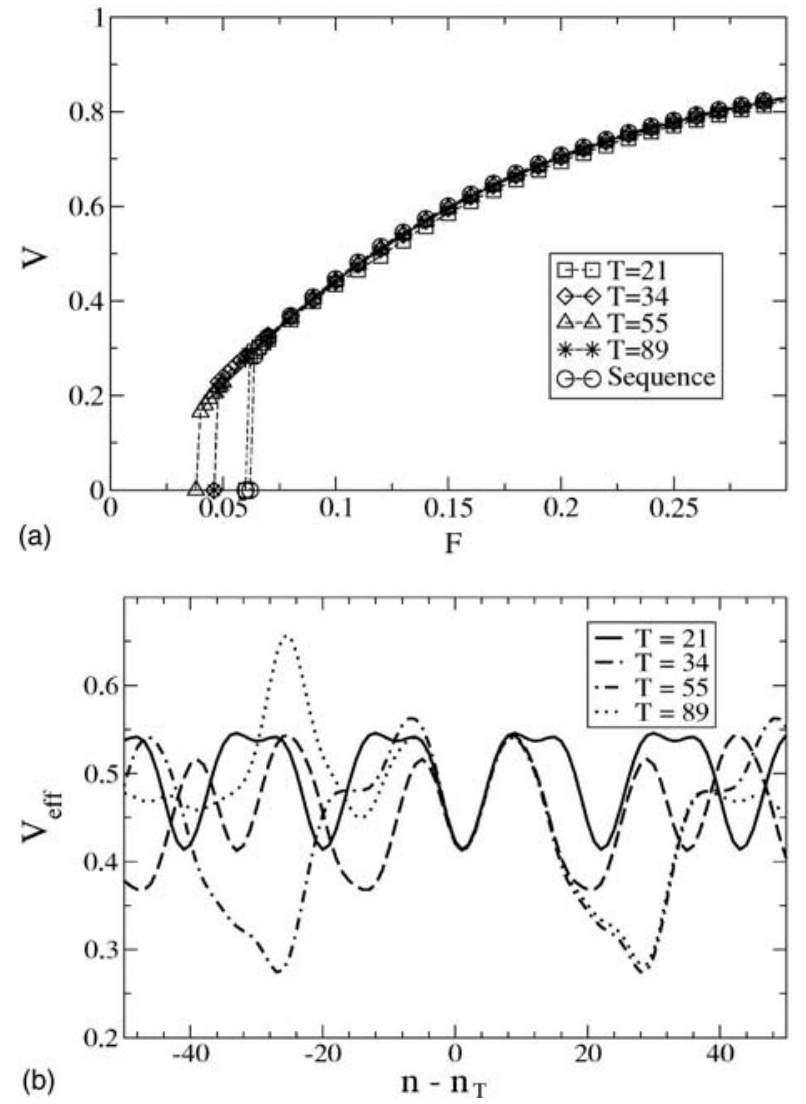

FIG. 8. (a) Velocity vs applied force for different periodic systems formed by repeating as a unit cell $n$ sites of the same contig as in Fig. 6 beginning at site 114 241, with $n=21,34,55$, and 89 (as in the Fibonacci case) and for the whole sequence going from 114100 to 115 100. (b) Effective potential for the systems in (a).

Having disregarded the relevance of the genetic information to the determination of the threshold force, it is interesting to proceed with the comparison between the previous work on the Fibonacci chain and the present results, in order to understand what is the difference and what leads to different conclusions. To this end, we have simulated periodic systems with a unit cell built from pieces of a genetic sequence, repeated to complete a longer chain, much as was done with the $F_{k}$ building blocks in [19] (see above). The sizes for the unit cells were chosen to mimic the sizes for the Fibonacci iterations, and again the starting points were chosen in the same position related to the beginning of a unit cell. The results are collected in Fig. 8(a). From this plot, we see that the most important difference between the two cases is that in the actual DNA chain, the threshold value does change much more for sequences with different unit cells than in the Fibonacci case, and there is not a maximum value for the threshold force when increasing the sizes of the unit cells.

One important point we want to stress is that the analytical approach in terms of collective coordinates and an effective potential is a very good picture of the observed phenomenology, even in this more realistic setting. In fact, the results in Fig. 8(a) can be correlated with the effective potentials depicted in Fig. 8(b). On the basis of this scenario, we suggest that the reason for the difference between the two mod- 
els lies in the larger diversity available for possible DNA chains. The fact that, in Fibonacci chains, sites of type $q_{b}$ are always isolated and that more than two sites of type $q_{a}$ cannot be found together is very restrictive as to the shapes and sizes of the peaks of wells of the potential felt by the kinks, giving rise to the Fibonacci structure mentioned in Sec. II C, and therefore the Fibonacci model cannot capture the richness of the DNA model. This is clearly seen when comparing the effective potential for the Fibonacci chains in Figs. 5(a) and 5(b) with that for the DNA chain in Fig. 8(b). On the other hand, when joining two unit cells in the DNA model, the local A-T/C-G concentration can vary more than in the Fibonacci case (where it is restricted to be close to a golden mean ratio), so a lower well or a higher peak may arise at the joining places. Finally, it is clear that considering longer and longer sequences of DNA increases the probability of finding longer repeated GC sequences, and hence of finding larger thresholds, as discussed above.

\section{CONCLUSIONS}

In this paper, we have reported the first conclusions of our work on the effects of the genetic sequence on the propagation of nonlinear excitations along (simple models of) DNA chains. The present stage of the research was motivated by previous results on chains given by aperiodic sequences [19], which led to the hypothesis that the information content of the inhomogeneities might be relevant for the dynamical behavior. While this general statement cannot be ruled out at this stage (at least from a probabilistic approach, as discussed above), we believe that at the level of the Englander model [10], the simulations presented here do not confirm that hypothesis. Specifically, we have not observed a qualitative difference in the dynamics of kinks propagating along coding or noncoding regions, which in principle prevents the use of this simple model as a genome sequencing tool. In this respect, it is important to realize that unzipping experiments cannot distinguish between the breaking of single base pairs, as discussed in [9]. Therefore, the fact that kink-pulling experiments or simulations do not discriminate between coding and noncoding regions would have a physical basis, because such discrimination would require a much better resolution. On the other hand, we are becoming increasingly aware that the function and information content of noncoding regions are far from well known, and it may well be that the dynamics in the two types of sequences is the same because the quantity of information is similar. Indeed, we are now beginning to realize that the noncoding regions do have crucial implications in the DNA-protein connections, either by inhibiting the fabrications of proteins from DNA or by switching on and off specific genes [30]. This is certainly an inter- esting issue that deserves further attention. In this regard, it would be interesting to come back to the model with artificially designed sequences, with different information content (in the Shannon-Kolmogorov sense), in order to settle definitely this question.

Even if the answer to the relation between behavior under applied forces and information content is finally negative, there is another conclusion arising from our research that can be relevant from the genomical viewpoint. We have verified that the effective potential approach $[19,26,28]$ to the dynamics of nonlinear excitations in DNA gives a very good description of the important points of the sequence, namely, valleys and peaks. Although the precise dynamics of kinks is difficult to predict, and specifically, the identification of the relevant valleys among all those present in the sequence is not yet understood, this analytical approximation allows us to identify the possible stop positions as well as the barriers that control the total opening (mechanical denaturation) of the chain. In fact, the complicated structure of the effective potential is consistent with the mechanical unzipping dynamics as observed in experiments [22], characterized by a stickslip behavior with large fluctuations in the velocity (also reproduced by our simple model). On the other hand, the comparison with the Fibonacci system suggests that the features of the effective potential that are most relevant for the dynamics are the highest peaks and the deepest valleys, the total potential landscape being responsible only for finer details. This is also true in the presence of thermal noise: Results from Langevin simulations show that very (in the scale of the applied forces) large temperatures do not change much the threshold values [31]. This suggests that the effective potential may be a way to identify promoter sequences much simpler than the full simulations reported in [25]. In addition, the effective potential can also be used as a tool in the gene identification procedure of Yeramian [17], which may lead to results of similar quality with much less computational effort. Of course, a detailed comparison with more complex models, such as the Peyrard-Bishop one [11-13], is needed to ascertain the usefulness of the effective potential picture. Work along these lines is in progress.

\section{ACKNOWLEDGMENTS}

We thank Michel Peyrard for many discussions about this work and for his patient explanations on DNA dynamics. We thank also Fernando Falo for discussions on the unzipping experiments. This work has been supported by the Ministerio de Ciencia y Tecnología of Spain through Grant No. BFM2003-07749-C05-01. S.C. is supported by the Consejería de Educación de la Comunidad Autónoma de Madrid and the Fondo Social Europeo. 
[1] A. C. Scott, Nonlinear Science (Oxford University Press, Oxford, 1999).

[2] E. Fermi, J. R. Pasta, and S. Ulam, Los Alamos Report LAUR-1940 (1955); reprinted in Collected Papers of Enrico Fermi, edited by E. Segré (University of Chicago Press, Chicago, 1965).

[3] A. S. Davydov, Phys. Scr. 20, 387 (1979).

[4] A. S. Davydov, Solitons in Molecular Systems, 2nd ed. (Kluwer, Dordrecht, 1991).

[5] L. V. Yakushevich, Q. Rev. Biophys. 26, 201 (1993).

[6] G. Gaeta, C. Reiss, M. Peyrard, and T. Dauxois, Riv. Nuovo Cimento 17, 1 (1994).

[7] L. V. Yakushevich, Nonlinear Physics of DNA (Wiley, Chichester, 1998).

[8] G. Gaeta, J. Biol. Phys. 24, 81 (1999).

[9] M. Peyrard, Nonlinearity 17, R1 (2004).

[10] S. W. Englander, N. R. Kallenbach, A. J. Heeger, J. A. Krumhansl, and A. Litwin, Proc. Natl. Acad. Sci. U.S.A. 77, 7222 (1980).

[11] M. Peyrard and A. R. Bishop, Phys. Rev. Lett. 62, 2755 (1989).

[12] T. Dauxois, M. Peyrard, and A. R. Bishop, Phys. Rev. E 47, R44 (1993).

[13] T. Dauxois and M. Peyrard, Phys. Rev. E 51, 4027 (1995).

[14] A. Campa and A. Giansanti, Phys. Rev. E 58, 3585 (1998).

[15] R. M. Wartell and A. S. Benight, Phys. Rep. 126, 67 (1985).

[16] A good summary of the experimental advances can be found in the collection of articles, The Double Helix-50 Years [Nature
(London) 421, 396 (2003)].

[17] E. Yeramian, Gene 255, 139 (2000); 255, 151 (2000); E. Yeramian, S. Bonnefoy, and G. Langsley, Bioinformatics 18, 190 (2002); E. Yeramian and L. Jones, Nucleic Acids Res. 31, 2843 (2003).

[18] G. Kalosakas, K. Ø Rasmussen, A. R. Bishop, C. H. Choi, and A. Usheva, e-print cond-mat/0309157 (2003); C. H. Choi, G. Kalosakas, K. Ø Rasmussen, M. Hiromura, A. R. Bishop, and A. Usheva, Nucleic Acids Res. 32, 1584 (2004).

[19] F. Domínguez-Adame, A. Sánchez, and Yu. S. Kivshar, Phys. Rev. E 52, R2183 (1995).

[20] C. R. Calladine and H. R. Drew, Understanding DNA, 2nd ed. (Academic Press, San Diego, 1997).

[21] D. W. McLaughlin and A. C. Scott, Phys. Rev. A 18, 1652 (1978).

[22] U. Bockelmann, B. Essevaz-Roulet, and F. Heslot, Phys. Rev. Lett. 79, 4489 (1997).

[23] M. Salerno, Phys. Rev. A 44, 5292 (1991).

[24] M. Salerno, Phys. Lett. A 167, 49 (1992).

[25] E. Lennholm and M. Hörnquist, Physica D 177, 233 (2003).

[26] M. Salerno and Yu. S. Kivshar, Phys. Lett. A 193, 263 (1994).

[27] A. Sánchez and A. R. Bishop, SIAM Rev. 40, 579 (1998).

[28] E. Lennholm and M. Hörnquist, Phys. Rev. E 59, 381 (1999).

[29] http://www ncbi nlm nih.gov.

[30] S. R. Eddy, Nat. Genet. 2, 919 (2001).

[31] S. Cuenda and A. Sánchez, Fluct. Noise Lett. (to be published), e-print q-bio/0403003. 